\title{
A cross sectional analysis of a case-control study about quality of life in CRS in the UK; a comparison between CRS subtypes*
}

\author{
Sally Erskine 1,2, Claire Hopkins ${ }^{3}$, Nirmal Kumar', Janet Wilson ${ }^{5}$, Allan Clark', \\ Alasdair Robertson ${ }^{6}$, Naveed Kara ${ }^{7}$, Vishnu Sunkaraneni ${ }^{8}$, Shahram Anari ${ }^{9}$, \\ Rhinology 54: 311-315, 2016 \\ Carl Philpott ${ }^{1,2}$, on behalf of the CRES Group \\ DOI:10.4193/Rhino15.36 \\ *Received for publication: \\ December 7, 2015 \\ ' Norwich Medical School, University of East Anglia, Norwich, UK \\ Accepted: March 31, 2016 \\ 2 ENT Department, James Paget University Hospital NHS Foundation Trust, Great Yarmouth, UK \\ ${ }^{3}$ ENT Department, Guy's and St Thomas' NHS Foundation Trust, London, UK \\ ${ }^{4}$ ENT Department, Writington, Wigan and Lee NHS Foundation Trust, Wigan, UK \\ ${ }^{5}$ Head \& Neck Surg. Institute of Health \& Society, Newcastle University, Newcastle, UK \\ ENT Department, Southern General Hospital, Glasgow, UK \\ ENT Department, Darlington Memorial Hospitals NHS Foundation Trust, Darlington, UK \\ ENT Department, Royal Surrey County Hospital, Surrey, UK \\ ${ }^{9}$ ENT Department, Heart of England NHS Foundation Trust, Birmingham, UK
}

\begin{abstract}
Background: The Sinonasal Outcome Test (SNOT-22) has been used as a patient reported outcome measure to grade symptom severity before and after treatment for chronic rhinosinusitis (CRS).

Methodology: This analysis uses data from the CRS Epidemiology Study (CRES). The overarching aim of CRES was to determine factors that influence the onset and severity of CRS. A study-specific questionnaire including SNOT-22 was distributed to patients with CRS attending ENT clinics across 30 centres in the United Kingdom. The aim of this analysis was to compare SNOT-22 scores between those with different types of CRS to determine any differences present in the total score or the subdomains and to assess whether any differences varied according to gender.
\end{abstract}

Results: There were a total of 1249 CRS participants in the following subgroups: CRS without nasal polyps (CRSsNPs) ( $n=553)$, CRS with nasal polyps (CRSwNPs) ( $n=651)$, allergic fungal rhinosinusitis (AFRS) $(n=45)$. Since there were differing gender ratios in each subgroup, males and females were analysed separately. The mean and standard deviation for SNOT-22 was: males CRSsNP 41.1 (21.0), CRSwNP 41.7 (20.5); females CRSsNP 49.6 (19.7), CRSwNP 49.5 (22.9). In the nasal domain, those with CRSwNP scored more highly than those with CRSsNP; for males 18.1 (8.1) vs. 15.9 (7.9); for females 19.6 (8.0) vs 16.7 (7.5).

Conclusions: Patients with CRSwNPs report higher symptom scores in the nasal domain of SNOT-22 than those with CRSsNPs with women in both subgroups reporting higher total scores than men.

Key words: quality of life, sinusitis, respiratory symptoms, nasal obstruction, facial pain, olfaction disorders

\section{Introduction}

Chronic rhinosinusitis (CRS) affects a significant proportion of the population, and as such is a burdensome disease to both individual sufferers and to the population as a whole ${ }^{(1)}$.
There are presently two accepted broad subcategories of CRS; CRSwNP, CRSsNPs ${ }^{(2)}$; with the more severe, refractory AFRS a subset of CRSwNPs ${ }^{(3)}$. Symptoms and their severity may vary widely between patients with CRS. Many questionnaires 
have been proposed to help quantify and evaluate patients' symptoms. The most widely accepted and best validated patient self-report symptom evaluation tool is the SNOT-22, whose 22 items incorporate both nasal and non-nasal symptoms ${ }^{(4)}$. It is a modification of the 31-question Rhinosinusitis Outcome Measure (RSOM-31) ${ }^{(5)}$ and an advancement of the SNOT-20 ${ }^{(6)}$. When used to monitor response to treatment the minimum clinically importance difference (MCID) is 9 points on the SNOT$22{ }^{(7)}$. Factor analysis has identified four principal SNOT subscales - nasal, facial, sleep and mood and we have considered these in our analysis ${ }^{(6,7-9)}$.

The overarching aim of the CRS Epidemiology Study (CRES) was to aid better understanding of medical and non-medical factors contributing to development or worsening of CRS. The aim of this analysis is to evaluate qualitative and quantitative differences in the SNOT-22 scale among different categories of rhinosinusitis in the substantial population of patients studied in the CRES.

\section{Materials and methods}

\section{Study design and setting}

The CRES was approved by the Oxford C Research Ethics Committee, sponsored by the University of East Anglia (UEA) and funded by the Anthony Long and Bernice Bibby Trusts. The study was conducted as a case-control questionnaire study and recruited from a total of 30 sites from around the UK (including the devolved nations of Wales and Scotland), between 2007 and 2013. The CRES questionnaire included study specific questions relating to socio-economic, environmental and medical comorbidity variables as well as the validated Short Form 36 (SF$36)^{(10)}$ quality of life measure and SNOT-22 ${ }^{(11)}$. Within SNOT-22, self-reported symptom severity is graded from $0-5$, with 5 being a severe problem. Scores for each question are added to produce an overall score of $0-1^{(11)}$. The subdomains used comprise the following questions; rhinological symptoms (blowing nose, sneezing, runny nose, nasal obstruction, loss of smell/taste), ear and facial symptoms (ear fullness, dizziness, ear pain, facial pain/ pressure), sleep function (difficulty falling asleep, waking up at night, lack of a good night's sleep) and psychological issues (fatigue, reduced productivity, reduced concentration, frustrate/restless/irritable, sad, embarrassed). The questions regarding cough and waking up tired were not included in these four subscales.

\section{Participants and methods}

The diagnosis of CRS was confirmed by an Otorhinolaryngologist according to the criteria below. Patients with CRS presenting to secondary/tertiary care ENT outpatient clinics were invited to participate in the study, regardless of symptom or disease severity or previous treatment, provided they conformed to the following criteria:

\section{Inclusion criteria:}

CRS with or without polyps as defined by the criteria laid out in EPOS $2012^{(2)}$. Symptoms must be present for at least 12 weeks and include:

- nasal blockage/obstruction/congestion and/or nasal discharge (anterior/posterior nasal drip)

- and either facial pain/pressure and/or reduction or loss of smell

and additionally:

- endoscopic signs of: polyps and/or mucopurulent discharge primarily from middle meatus and/or; oedema/mucosal obstruction primarily in middle meatus

- and/or CT changes: mucosal changes within the ostiomeatal complex and/or sinuses

Any patients with nasal polyps placed in the AFRS category met the Bent and Kuhn criteria ${ }^{(11)}$ or the St Paul's Sinus Centre modification of this ${ }^{(12)}$.

Patients and controls included were at least 18 years of age.

\section{Exclusion criteria:}

- Patients unable to comprehend written English.

All participants were provided with information leaflets but as the study specific questionnaire was anonymous, consent was implied through participation. Questionnaires were returned by participants using a freepost envelope and scanned to a secure database using Formic. Two members of the research team checked the accuracy of electronic scanning of returned questionnaires against the original copy.

\section{Statistical analysis}

Since there were differing gender ratios in each subgroup, males and females were analysed separately. Descriptive analysis was undertaken with the mean and standard deviation reported for continuous variables and the number and percentage for categorical variables. Due to small numbers in the AFRS group, it was decided that those with AFRS would be included in the CRSwNPs group. The SNOT-22 score and the principal subscales were compared using independent samples t-tests for compare the means between individuals with CRSsNPS and CRSwNPS. Due to the difference in ages between these groups they were always adjusted for age using a linear regression model with age as a predictor variable.

\section{Results}

A total of 1249 completed CRES questionnaires from CRS patients were available for analysis (Table 1). Detailed description of the geographical distribution of participants has been published previously ${ }^{(13)}$. There were no significant differences in total SNOT-22 score between disease subtypes amongst either males or females (Table 2). There were significant differences in nasal symptom domain scores between those with and without 
Table 1. Summary of participants submitting SNOT-22 questionnaires.

\begin{tabular}{lcccc} 
Participants & $\begin{array}{c}\text { Controls } \\
\mathbf{N = 2 2 1}\end{array}$ & $\begin{array}{c}\text { CRSsNP } \\
\mathbf{5 5 3}\end{array}$ & $\begin{array}{c}\text { CRSwNP } \\
\mathbf{6 5 1}\end{array}$ & $\begin{array}{c}\text { AFRS } \\
\mathbf{4 5}\end{array}$ \\
\hline Females & $143(68.4 \%)$ & $259(53.1 \%)$ & $185(32.2 \%)$ & $19(43.2 \%)$ \\
$\begin{array}{l}\text { Mean Age } \\
\text { (SD) }\end{array}$ & $47.3(14.9)$ & $51.8(15.3)$ & $56.0(14.6)$ & $56.1(12.7)$ \\
Range & $19-82$ & $18-84$ & $17-102$ & $20-76$ \\
\hline
\end{tabular}

polyps, with those with CRSwNPs having the highest scores, this difference existed amongst both males and females. For the facial and emotional domains, those with CRSsNPs scored more highly than those with CRSwNPs and this tended towards significance in the facial domain for females.

Whilst the analysis was separated for gender for the reason cited above, it is worth noting that women with and without polyps scored significantly more highly than males on the SNOT-22: mean of 49.6 vs 41.1 for CRSsNP and 49.5 vs 41.7 for CRSwNP. Such differences are clinically significant since they are 8.5 and 8.4 respectively, close to the minimum clinically important difference of 8.9 points ${ }^{(11)}$.

\section{Discussion}

Our study has evaluated SNOT-22 scores across a large and diverse population and has found that there are significant differences in the nasal domain between those in the two main subgroups of CRS. In a disease such as CRS which consists of multiple and variable symptoms, SNOT-22 scores enable us to assess the global impact of disease on a patient, and if repeated at intervals provides a comparator over time which may be used to assess response to treatment. These are important considerations when measuring outcomes. In contrast to objective measures, such as CT scans, peak nasal inspiratory flow or smell testing, which measure only a single symptom or measure of nasal function, SNOT-22 provides a more comprehensive assessment of disease burden through global symptoms and quality of life impact. By showing differences in CRS symptoms in different subtypes of CRS we may be able to better understand and quantify disease severity.

The SNOT-22 score has already been shown to be a useful predictor of the improvement in QoL that could be expected after sinus surgery for CRS ${ }^{(14,15)}$. Tan et al. found that the frequency of individual symptoms varied the likelihood of a CRS diagnosis and consequently varied the most effective management algorithm to choose ${ }^{(16)}$; a more detailed understanding of SNOT22 in different disease subtypes may further inform clinical decision making. A study of 126 patients by Banerji et al. using 'SNOT-20+1' found similar differences ${ }^{(17)}$; nasal obstruction and hyposmia/anosmia were more prevalent in those with polyps and facial pain/pressure/headache were more prevalent in those without. Using the Rhinosinusitis Symptom Inventory (RSI), Bhattacharyya also found higher scores for nasal symptoms in those with polyps and higher scores for facial symptoms in those without ${ }^{(18)}$. In a study of 234 patients, Dietz de Loos et al. used the Rhinosinusitis Outcome Measure 31 (RSOM 31) and found that those with polyps were more likely to score highly on nasal symptoms compared to those without polyps ${ }^{(19)}$. This observation therefore appears consistent regardless of the PROM used, and may be due to the physical impact of nasal polyps filling the nasal cavity in those with CRSwNPs. Since our study was anonymous, it was not possible to correlate our scores with

Table 2. Total SNOT-22 and domain scores for all subtypes.

\begin{tabular}{|c|c|c|c|c|c|c|}
\hline & \multirow[t]{2}{*}{ CRSsNP } & \multirow[t]{2}{*}{ CRSwNPs } & \multicolumn{2}{|c|}{ Unadjusted } & \multicolumn{2}{|c|}{ Adjusted for age } \\
\hline & & & $\begin{array}{l}\text { Mean difference } \\
(95 \% \mathrm{Cl})\end{array}$ & p-value & $\begin{array}{c}\text { Mean difference } \\
(95 \% \mathrm{Cl})\end{array}$ & p-value \\
\hline \multicolumn{7}{|l|}{ Males only } \\
\hline SNOT-22 & $41.1(21.0)$ & $41.7(20.5)$ & $0.60(-2.87,4.08)$ & 0.7328 & $1.23(-2.28,4.75)$ & 0.490 \\
\hline Nasal & $15.9(7.9)$ & $18.1(8.1)$ & $2.20(0.87,3.53)$ & 0.0012 & $2.43(1.07,3.79)$ & $<0.001$ \\
\hline Facial & $5.0(4.5)$ & $4.3(4.1)$ & $-0.73(-1.44,-0.02)$ & 0.0453 & $-0.55(-1.26,0.17)$ & 0.134 \\
\hline Sleep fatigue & $5.8(4.3)$ & $6.1(4.3)$ & $0.26(-0.45,0.98)$ & 0.4661 & $0.32(-0.41,1.05)$ & 0.387 \\
\hline Emotion & $9.7(7.2)$ & $9.2(7.1)$ & $-0.50(-1.69,0.69)$ & 0.4055 & $-0.39(-1.60,0.81)$ & 0.520 \\
\hline \multicolumn{7}{|l|}{ Females only } \\
\hline SNOT-22 & $49.6(19.7)$ & 49.5 (22.9) & $-0.12(-4.16,3.91)$ & 0.9518 & $1.03(-3.07,5.13)$ & 0.622 \\
\hline Nasal & $16.7(7.5)$ & $19.6(8.0)$ & $2.86(1.40,4.31)$ & 0.0001 & $3.19(1.70,4.67)$ & $<0.001$ \\
\hline Facial & $7.4(4.9)$ & $6.3(4.7)$ & $-1.09(-2.01,-0.18)$ & 0.0196 & $-0.88(-1.80,0.05)$ & 0.064 \\
\hline Sleep fatigue & $7.4(4.2)$ & $7.5(4.7)$ & $0.11(-0.71,0.93)$ & 0.7859 & $0.19(-0.65,1.04)$ & 0.656 \\
\hline Emotion & $12.7(7.4)$ & $11.1(7.9)$ & $-1.64(-3.08,-0.21)$ & 0.0251 & $-1.10(-2.55,0.35)$ & 0.137 \\
\hline
\end{tabular}


any objective measures of severity such as Lund-Mackay score, although previous studies have found only a weak association between preoperative SNOT-22 scores and Lund-Mackay scores (20,21).

Amongst females, the difference in the facial domain between those with CRSsNPs and those with CRSwNPs approached significance. The reasons behind this difference are equally complex but our findings are supported by previous work which found that CRSsNPs had more impact on vitality and bodily pain than did CRSwNPs ${ }^{(22)}$. We know from our own cohort that rates of consultation with a General Practitioner for depression and anxiety are higher amongst those with CRSsNPs ${ }^{(23)}$, but the direction of this association is difficult to establish. It may be that the underlying autonomic driver behind symptom generation is greater in patients with CRSsNPs; both state and trait anxiety have been found to be higher amongst those with both allergic rhinitis and vasomotor rhinitis than controls ${ }^{(24)}$. Symptom generation may therefore interact with ANS dysfunction. This reflects the wide-ranging impact of CRS on patients over and above purely nasal symptoms. Gender differences are to be expected; previous literature including the UK Sino nasal audit data ${ }^{(25)}$ suggests that females are more likely to report somatic symptoms ${ }^{(26)}$ including nasal symptoms ${ }^{(27)}$. We have shown that in addition to higher scores overall, the composition of scores may also be different.

\section{Clinical uses}

The strength of PROMs such as SNOT-22 lies in the fact that they are not subject to individual clinicians' interpretation and can be used by patients to chart the course of their disease between primary and secondary care and before and after any intervention. There has been debate as to whether diagnosis according to questionnaire adequately correlates to clinical diagnosis by a clinician. Lange et al. investigated this dilemma and found moderate agreement between the questionnaire and clinician based diagnosis ${ }^{(21)}$. So whilst using SNOT-22 in combination with traditional clinical assessment including nasendoscopy, as in this study, is likely to be the best route to accurate diagnosis, SNOT-22 could be a useful tool for General Practitioners when making decisions regarding treatment response and/or onward referral depending on symptom severity, even in the absence of nasendoscopy. This may avoid delays in treatment escalation and referral which are known to be harmful for patients when they have no benefit from first-line therapies ${ }^{(28)}$. However, the SNOT-22 may be challenging to complete in a short GP appointment; the nasal domain may be useful in this regard as it would be quick to administer and repeat. Equally, if General Practitio- ners using SNOT-22 note that patients score highly in the nonnasal domains, such as sleep, further questions as to the nature of such symptoms may result in more holistic management through non-nasal interventions, such as treatment of related anxiety and depression ${ }^{(23)}$.

The distribution of scores for those with and without polyps was too broad to allow identification of different phenotypes according to SNOT-22 score alone, but greater knowledge and use of the sub-domains may help guide differentiation between both controls and different subgroups, and may allow better correlation with more traditional objective measures. The predictive value of the domains requires further evaluation. SNOT-22 score could further refine currently used diagnostic criteria to quantify subjective interpretations of patients' symptoms ${ }^{(2)}$.

\section{Conclusion}

Our analysis has found significant differences in symptom reporting between CRSsNPs and CRSwNPs. Whilst this principle is supported by previous studies, our research has shown for the first time that such differences are represented by significant differences in the nasal domain of SNOT-22. PROMs are increasingly important in clinical care and research; this finding aids understanding into the way SNOT-22 score is composed amongst patients with different CRS subtypes and may help in understanding differing treatment responses for these patient groups as quantified by SNOT-22. Our study has also shown differences in symptom reporting between males and females and is the largest UK study to quantify these differences amongst different CRS subtypes. Gender differences therefore should be considered when researching and treating CRS. Evaluation of symptoms in combination with endoscopic examination and/or CT scanning remains the gold standard for diagnosis but SNOT22 is important in evaluating patients' experiences of symptoms and changes over time. Future work may include development of a utility tool based on the SNOT-22 that may be used more effectively in a primary care setting to help select appropriate treatment and referral pathways.

\section{Authorship contribution}

$\mathrm{SE}, \mathrm{CP}$ : Design of study, data collection, data analysis, writing of paper; $\mathrm{CH}, \mathrm{NKu}, \mathrm{AR}, \mathrm{NKa}, \mathrm{VS}, \mathrm{SA}$ : data collection, data analysis, writing of paper; JW: analysis, writing of paper; AC: design, statistics, writing of paper. The CRES Group - Principle Investigators at sites across UK responsible for running of the study locally

\section{Conflict of interest}

No conflict of interest for any author identified. 


\section{References}

1. Hastan D, Fokkens WJ, Bachert C, et al. Chronic rhinosinusitis in Europe - an underestimated disease. A GA2LEN study. Allergy. 2011; 66: 1216-23.

2. Fokkens WJ, Lund VJ, Mullol J, et al. EPOS 2012: European position paper on rhinosinusitis and nasal polyps 2012. A summary for otorhinolaryngologists. Rhinology. 2012 50: 1-12.

3. Kuhn FA, Swain Jr R. Allergic fungal sinusitis: diagnosis and treatment. Curr Opin Otolaryngol Head Neck Surg. 2003; 11: 1-5.

4. Hopkins C, Gillett S, Slack R, Lund VJ, Browne JP. Psychometric validity of the 22-item Sinonasal Outcome Test. Clin Otolaryngol. 2009; 34: 447-54

5. Piccirillo JF, Edwards D, Haiduk A, Yonan C Thawley SE. Psychometric and Clinimetric Validity of the 31-Item Rhinosinusitis Outcome Measure (RSOM-31). Am J Rhinol. 1995; 9: 297-306.

6. Piccirillo JF, Merritt MG, Jr., Richards ML. Psychometric and clinimetric validity of the 20-Item Sino-Nasal Outcome Test (SNOT20). Otolaryngol Head Neck Surg. 2002; 126: 41-7.

7. DeConde AS, Mace JC, Bodner T, et al. SNOT-22 quality of life domains differentially predict treatment modality selection in chronic rhinosinusitis. Int Forum Allergy Rhinol. 2014; 4: 972-9.

8. Lange B, Holst R, Thilsing T, Baelum J and Kjeldsen A. Quality of life and associated factors in persons with chronic rhinosinusitis in the general population: a prospective questionnaire and clinical cross-sectional study. Clin Otolaryngol. 2013; 38: 474-80.

9. Lange B, Thilsing T, Al-kalemji A, Baelum J, Martinussen T, Kjeldsen A. The Sino-Nasal Outcome Test 22 validated for Danish patients. Dan Med Bull. 2011; 58

10. McHorney CA, Ware JE, Jr., Lu JF, Sherbourne CD. The MOS 36-item Short-Form Health Survey (SF-36): III. Tests of data quality, scaling assumptions, and reliability across diverse patient groups. Med Care. 1994; 32: 40-66.
11. Bent JP, 3rd, Kuhn FA. Diagnosis of allergic fungal sinusitis. Otolaryngol Head Neck Surg. 1994; 111: 580-8.

12. Philpott $C M$, Javer $A R$, Clark A. Allergic fungal rhinosinusitis - a new staging system. Rhinology. 2011; 49: 318-23.

13. Philpott C, Erskine S, Hopkins C, Coombes E, Kara N, Sunkareneni V, Anari S, Salam M, Farboud A, Clark A. A case-control study of medical, psychological and socio- economic factors in uencing the severity of chronic rhinosinusitis. Rhinology. 2016; 54: 134-140.

14. Hopkins C, Rudmik L, Lund VJ. The predictive value of the preoperative Sinonasal outcome test-22 score in patients undergoing endoscopic sinus surgery for chronic rhinosinusitis. Laryngoscope. 2015; 125: 1779-84.

15. Rudmik L, Soler ZM, Mace JC, Schlosser RJ, Smith TL. Economic evaluation of endoscopic sinus surgery versus continued medical therapy for refractory chronic rhinosinusitis. Laryngoscope. 2015; 125: 25-32.

16. Tan BK, Lu G, Kwasny MJ, et al. Effect of symptom-based risk stratification on the costs of managing patients with chronic rhinosinusitis symptoms. Int Forum Allergy Rhinol. 2013; 3: 933-40.

17. Banerji A, Piccirillo JF, Thawley SE, et al. Chronic rhinosinusitis patients with polyps or polypoid mucosa have a greater burden of illness. Am J Rhinol. 2007; 21: 19-26.

18. Bhattacharyya N. Clinical and Symptom Criteria for the Accurate Diagnosis of Chronic Rhinosinusitis. Laryngoscope. 2006; 116: 1-22

19. Dietz de Loos DAE, Hopkins C, Fokkens WJ. Symptoms in chronic rhinosinusitis with and without nasal polyps. Laryngoscope. 2013; 123: 57-63.

20. Hopkins C, Browne JP, Slack R, Lund VJ, Brown P. The Lund-Mackay staging system for chronic rhinosinusitis: how is it used and what does it predict? Otolaryngol Head Neck Surg. 2007; 137: 555-61.

21. Lange B, Thilsing T, Baelum J, Holst R, Kjeldsen A. Diagnosing chronic rhinosinusitis: comparing questionnaire-based and clinical-based diagnosis. Rhinology. 2013; 51: 128-36.

22. Durr DG, Desrosiers MY and Dassa C. Impact of rhinosinusitis in health care delivery: the Quebec experience. Journal of Otolaryngology. 2001; 30: 93-7.

23. Erskine S, Philpott CM. Chronic Rhinosinusitis and Mood Disturbance. Otolaryngol Head Neck Surgery. 2014; 151: P114.

24. Addolorato G, Ancona C, Capristo E, et al State and trait anxiety in women affected by allergic and vasomotor rhinitis. Journal of Psychosomatic Research. 1999; 46: 283-9.

25. Hopkins C, Gillett S and Slack R. Are men really more full of SNOT? : Clin Otolaryngol. 2009 Jun:34(3):267-8.

26. Barsky AJ, Peekna HM and Borus JF. Somatic Symptom Reporting in Women and Men. J Gen Int Med. 2001; 16: 266-75.

27. Rydn O, Andersson B and M. A. Disease perception and social behaviour in persistent rhinitis:a comparison between patients with allergicand nonallergic rhinitis. Allergy. 2004; 59: 461-4.

28. Erskine SE, Verkerk MM, Notley C, Williamson IG and Philpott CM. Chronic Rhinosinusitis: Patient Experiences of Primary and Secondary Care - A Qualitative Study. Clin Otolaryngol. 2016;41(1):8-14.

Sally E. Erskine

c/o C. Philpott

Norwich Medical School

University of East Anglia

Norwich Research Park

Norwich, NR4 7TJ

United Kingdom

E-mail: sally.erskine@doctors.org.uk 